\title{
Design of Patient-Specific Gait Modifications for Knee Osteoarthritis Rehabilitation
}

\author{
Benjamin J. Fregly*, Jeffrey A. Reinbolt, Kelly L. Rooney, Kim H. Mitchell, and Terese L. Chmielewski
}

\begin{abstract}
Gait modification is a nonsurgical approach for reducing the external knee adduction torque in patients with knee osteoarthritis (OA). The magnitude of the first adduction torque peak in particular is strongly associated with knee $\mathrm{OA}$ progression. While toeing out has been shown to reduce the second peak, no clinically realistic gait modifications have been identified that effectively reduce both peaks simultaneously. This study predicts novel patient-specific gait modifications that achieve this goal without changing the foot path. The modified gait motion was designed for a single patient with knee OA using dynamic optimization of a patient-specific, full-body gait model. The cost function minimized the knee adduction torque subject to constraints limiting how much the new gait motion could deviate from the patient's normal gait motion. The optimizations predicted a "medial-thrust" gait pattern that reduced the first adduction torque peak between $32 \%$ and $54 \%$ and the second peak between $34 \%$ and $56 \%$. The new motion involved three synergistic kinematic changes: slightly decreased pelvis obliquity, slightly increased leg flexion, and slightly increased pelvis axial rotation. After gait retraining, the patient achieved adduction torque reductions of $39 \%$ to $50 \%$ in the first peak and $37 \%$ to $55 \%$ in the second one. These reductions are comparable to those reported after high tibial osteotomy surgery. The associated kinematic changes were consistent with the predictions except for pelvis obliquity, which showed little change. This study demonstrates that it is feasible to design novel patient-specific gait modifications with potential clinical benefit using dynamic optimization of patient-specific, full-body gait models. Further investigation is needed to assess the extent to which similar gait modifications may be effective for other patients with knee OA.
\end{abstract}

Index Terms - Dynamic optimization, knee adduction moment, movement prediction, osteoarthritis (OA).

Manuscript received May 5, 2006; revised December 15, 2006. This work was supported in part by the Whitaker Foundation and in part by the National Institutes of Health (NIH) National Library of Medicine under Grant R03-LM07332. Asterisk indicates corresponding author.

*B. J. Fregly is with the Departments of Mechanical and Aerospace Engineering, Biomedical Engineering, and Orthopaedics and Rehabilitation, University of Florida, Gainesville, FL 32611 USA (e-mail: fregly@ufl.edu).

J. A. Reinbolt was with the the Department of Mechanical and Aerospace Engineering, University of Florida, Gainesville, FL 32611 USA. He is now with the Department of Bioengineering, Stanford University, Stanford, CA 94305 USA (e-mail: reinbolt@ stanford.edu)..

K. L. Rooney is with the Department of Biomedical Engineering, University of Florida, Gainesville, FL 32611 USA.

K. H. Mitchell is with the Biomotion Foundation, Palm Beach, FL 33480 USA.

T. L. Chmielewski is with the Department of Physical Therapy, University of Florida, Gainesville, FL 32611 USA (e-mail: tchmiele@ phhp.ufl.edu).

This paper contains supplementary multimedia material available at http:// ieeexplore.ieee.org, provided by the author. The material consists of videos in avi format.

Digital Object Identifier 10.1109/TBME.2007.891934

\section{INTRODUCTION}

D ESPITE the need for early treatment, few clinical interventions slow the progression of knee osteoarthritis (OA) and minimize future functional limitations. One of the most conservative surgical interventions is high tibial osteotomy (HTO). During this procedure, a wedge of bone is typically added to or removed from the proximal tibia, changing the alignment of the leg from bow legged to slightly knock kneed. The change in limb alignment is intended to shift some of the contact load from the diseased medial to the healthy lateral compartment of the knee. Since articular cartilage is responsive to the magnitude of joint loading, reducing compressive loads in the diseased compartment may slow the rate of cartilage breakdown and delay the need for joint replacement.

Since medial compartment load cannot be measured noninvasively in vivo, researchers have sought an external measure to quantify the load reduction achieved by HTO surgery. The best candidate found thus far is the external knee adduction torque during gait [1], [2]. This torque exhibits two peaks during the gait cycle - one during early stance and the other during late stance. Only the first peak during early stance has been shown to be higher in patients with knee OA compared to healthy controls [3]-[5], making the first peak the most critical one to lower. Following HTO surgery, patients with the lowest first peak tend to have the best long-term outcome [6], [7], particularly those whose peak is below approximately $2.5 \%$ body weight times height $(\% \mathrm{BW} * \mathrm{HT})$ [8]. Such patients typically obtain adduction torque reductions on the order of $30 \%$ to $50 \%$ from HTO surgery [3], [6], [9]. A high-peak knee adduction torque has also been correlated with higher proximal tibial bone density on the medial side [10], increased OA disease severity [11], and an increased rate of OA disease progression [12].

A noninvasive early treatment option that has received limited attention is gait modification to decrease the peak knee adduction torque. Ideally, if simple gait modifications could reduce the peak adduction torque by as much as HTO surgery, then the benefits of the surgery could be made available to a broad clinical population without the risks and costs of an invasive procedure. To date, at least five basic gait modifications have been shown to reduce the adduction torque in patients with knee OA: toeing out, walking more slowly, walking with decreased stride length, walking with increased medial-lateral trunk sway, or using lateral heel wedges. Walking with the toes pointed outward can reduce the second peak of the adduction torque curve by as much as $40 \%$ but has little influence on the first peak [2], [4], [13], [14]. Walking slower or with decreased stride length can reduce both peaks significantly in some patients but not others [15], but the speed or stride length decrease that is required to 
achieve a significant reduction may be larger than many patients would tolerate. While increasing medial-lateral trunk sway can reduce the first peak by up to $66 \%$ [16], the resulting gait motion does not look normal, which could deter many patients from adopting it. Recent gait studies using lateral heel wedges have reported only modest reductions in the peak adduction torque [17], [18]. Thus, no clinically realistic gait modifications have been identified that will reduce the first peak or both peaks of the knee adduction torque curve to an extent comparable to HTO surgery.

This feasibility study seeks to design a novel yet "normal looking" gait motion that reduces both adduction torque peaks to the same extent as HTO surgery but without changing the foot path or trunk orientation. The design process was tailored to an individual patient, utilized dynamic optimization of a patient-specific full-body gait model, and required the patient's pretreatment gait data as a starting point. The optimization results were used to teach the patient how to walk differently to reduce both adduction torque peaks simultaneously. Gait data collected from the patient following gait retraining were used to evaluate the extent to which the predicted modifications can be achieved and sustained in clinical practice.

\section{MATERIALS AND METHODS}

\section{A. Experimental Data Collection}

Gait data were collected from a single highly functional knee OA patient (male, age 37 years, height $170 \mathrm{~cm}$, mass $69 \mathrm{~kg}$, alignment $5^{\circ}$ varus with Kellgren and Lawrence grade 2 medial OA in both knees based on radiographic assessment). The subject gave informed consent and performed all experimental trials while wearing New Balance 608 sneakers. Three-dimensional (3-D) surface marker data using the Cleveland Clinic marker set with additional markers on the feet were collected at $120 \mathrm{~Hz}$ using a six-camera video-based motion analysis system (Motion Analysis Corporation, Santa Rosa, CA). The 3-D residuals from the camera calibration trial averaged $1.1 \mathrm{~mm}$ with a standard deviation of $0.5 \mathrm{~mm}$. Individual markers were located on both wrists, elbows, and shoulders, with three markers per segment placed on the pelvis and each thigh, shank, and hindfoot [19]. A static trial with additional markers over anatomical landmarks was performed to define segment coordinate systems and marker locations within those coordinate systems. Dynamic joint motion trials were performed for the hip, knee, and ankle to exercise their primary functional axes for determination of joint positions and orientations in the segment coordinate systems [19]. For the gait trials, the subject walked along a 15-m runway at a self-selected speed of $1.4 \mathrm{~m} / \mathrm{s}$, which was measured using the progression of the pelvis markers over the time between successive left heel strikes. Ground reaction forces and torques under each foot were measured at $1000 \mathrm{~Hz}$ around the electrical centers of two force plates (Advanced Mechanical Technology, Inc., Watertown, MA). The raw marker data were filtered using a fourth-order, zero phase-shift, low-pass Butterworth filter with a cutoff frequency of $6 \mathrm{~Hz}$. The data collection and subsequent computer simulations were approved by the institutional review board.
One complete gait cycle (left heel strike to left heel strike) without surface marker dropout was selected as the nominal data set for use in the optimization study. Due to the limitation of only two force plates, no ground reaction data were available for the right leg at the start of the cycle (i.e., the right leg was in contact with the ground, but not on a force plate, at the initial left heel strike). For the initial time frames when no right ground reaction data were available, the patient-specific full-body gait model developed for the optimization studies was used to estimate the right ground reactions from the left ones. Since only the left leg had experimental ground reaction data for the entire cycle, results from the subsequent optimization studies are presented only for that leg.

After optimization predictions were developed using the patient's nominal gait data, the patient attempted to train himself to produce the predicted gait motion on a qualitative basis. Training consisted of studying plots of the optimized kinematics, kinetics, center of pressure, and ground reactions, as well as animations comparing the nominal and optimal gait motions. The patient implemented the general kinematic alterations in a manner that was as natural and easy to achieve as possible compared to his original gait motion. Over a nine-month self-training period, the patient sought to incorporate these gait modifications into his normal walking pattern by constantly reminding himself to walk the new way, the goal being for the new gait motion to become second nature. After this period, the patient gave informed consent and was retested wearing the same sneakers. Testing was performed at a self-selected walking speed, which again was $1.4 \mathrm{~m} / \mathrm{s}$, under two conditions. First, the patient was asked to try to walk using his old gait motion prior to retraining, and second, he was asked to walk using an exaggerated version of the new gait motion predicted by the optimizations. These two walking patterns were selected to bound the experimental adduction torque changes that the patient could achieve as a result of the gait retraining process.

\section{B. Dynamic Model Development}

A parametric, 3-D, dynamic gait model was developed using two separate software packages. The first was Autolev (Online Dynamics, Inc., Sunnyvale, CA), a symbolic manipulator for deriving dynamical equations, and the second was Software for Interactive Musculoskeletal Modeling (SIMM) with the Dynamics Pipeline (Motion Analysis Corporation, Santa Rosa, CA). The Autolev model provided direct access to the symbolic form of the equations of motion, making it well suited for calibrating joint and inertial parameters to the patient's nominal gait data. In contrast, the SIMM/Pipeline model provided a well-structured dynamic simulation environment along with the ability to visualize the predicted motions and ground reaction force vectors using a skeletal model scaled to the patient's dimensions. Having two versions of the same dynamic model also permitted validation of the inverse dynamic analyses used in the optimization studies.

The gait model possessed 27 degrees of freedom (DOFs) composed of gimbal (3 DOFs), universal (2 DOFs), and pin (1 DOF) joints (Fig. 1). Similar to the model structure in [20], 


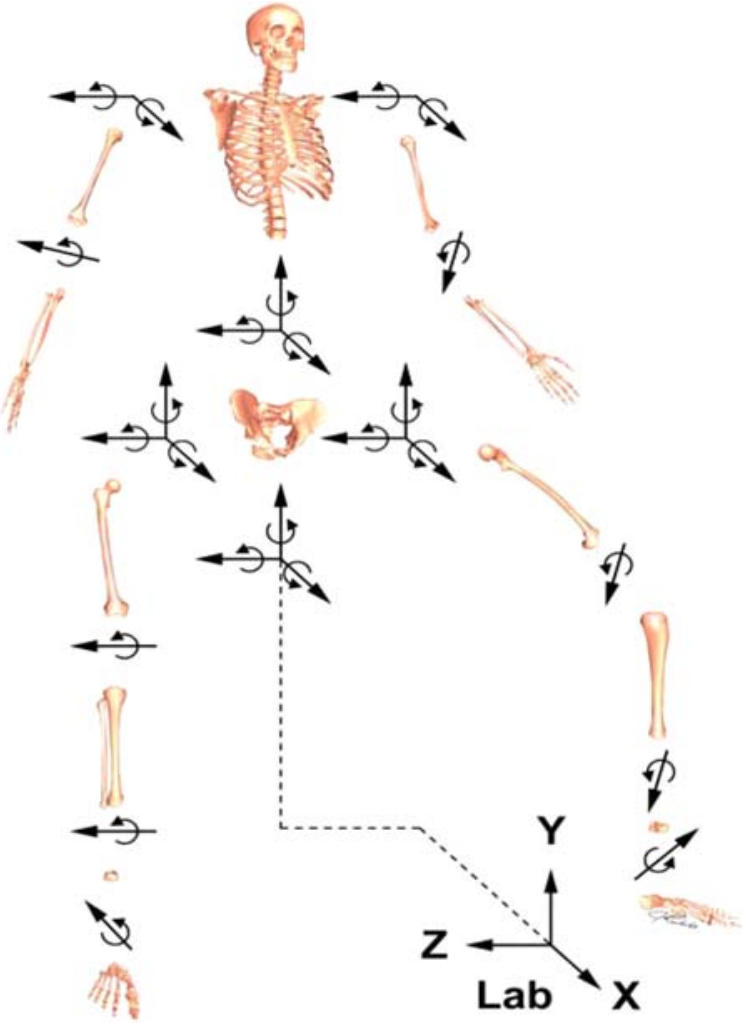

Fig. 1. Schematic of the 27 degree-of-freedom (DOF) full-body gait model used to predict novel gait motions that reduce the peak knee adduction torque. All joints are traditional engineering joints (gimbal, universal, pin) with the exception of the ground to pelvis joint which possesses 6 DOFs. Parameters defining the positions and orientations of joints in the body segments and the masses, mass centers, and moments of inertia of the body segments were calibrated to gait data collected from a single patient with knee osteoarthritis.

three translational and three rotational DOFs defined the position and orientation of the pelvis in the laboratory reference frame. For the lower body joints, each hip was modeled as a gimbal joint, each knee as a pin joint, and each ankle as two nonintersecting and nonorthogonal pin joints [21]. For the upper body, the back was modeled as a gimbal joint, each shoulder as a universal joint, and each elbow as a pin joint. The axes of each segment coordinate system were assumed to coincide with the central principal axes of the segment [22]. In lieu of a deformable ground contact model utilizing springs and dampers [22], [23], the ground reaction forces and torques calculated using the force plate electrical centers were treated as external loads applied to the feet during periods when the foot was known to be in contact with the ground [24]. This approach also eliminated the need for a toes segment in each foot model.

Inverse dynamic analyses were performed using the state-space form of the equations of motion from both full-body gait models. Consequently, 27 control forces and torques were calculated from the 27 equations of motion and the experimentally determined joint kinematics and ground reactions. The forces and torques calculated by the two models were identical to within roundoff error, providing a check on the equations of motion. The external knee adduction torque for each leg was calculated as the negative of the internal reaction torque about an axis directed anteriorly through the origin of the tibial coordinate system (i.e., midpoint between the femoral epicondyles). External (i.e., residual) forces and torques acting on the pelvis were calculated from the 6 DOF joint between the ground and pelvis. Since no external loads act on the pelvis in real life, nonzero external force or torque components at any time frame represent error in the model structure, model parameter values, and/or experimental data.

\section{Model Parameter Calibration}

Joint and inertial parameters in the model were calibrated to match the nominal gait data as close as possible. Calibration was performed using the Levenberg-Marquardt nonlinear least-squares optimization algorithm in Matlab (The Mathworks, Natick, MA) along with an Autolev version of the equations of motion that included the locations of the surface markers in the segment coordinate systems. The cost function simultaneously minimized errors between model and experimental marker locations in the laboratory reference frame, external pelvis forces and torques, and changes in inertial parameter values away from their initial values. The design variables were joint parameters (i.e., joint positions and orientations in the segment coordinate systems) for joints that underwent large $\left(>25^{\circ}\right)$ rotations [25], all inertial parameters (i.e., masses, mass centers, and moments of inertia), and polynomial and Fourier coefficients defining the trajectory of each joint translation and rotation [26]. Initial guesses for all joint parameters (hip, knee, and ankle on each side) were calculated from optimization of the isolated joint trial data [19], while initial guesses for all inertial parameters were calculated from [27].

Each optimizer function evaluation involved an inverse dynamics analysis performed with the Autolev version of the model over all time frames for which experimentally measured ground reaction forces and torques were available (or known to be zero) for both feet. To reach the optimal solution, joint and inertial parameters required relatively small changes from their initial guesses, at most $0.005 \mathrm{~m}$ for joint positions, $3.6^{\circ}$ for joint orientations, $0.57 \mathrm{~kg}$ for segment masses, $0.019 \mathrm{~m}$ for segment mass centers, and $0.079 \mathrm{~kg} \mathrm{~m}^{2}$ for segment inertias. The resulting root-mean-square (rms) errors were $0.013 \mathrm{~m}$ in surface marker positions and $24.5 \mathrm{~N}$ and $3.5 \mathrm{Nm}$ in external pelvis forces and torques, respectively.

\section{Dynamic Optimization Predictions}

We used an inverse dynamic optimization approach to design "normal looking" gait motions capable of reducing both adduction torque peaks simultaneously. In this context, "normal looking" means a gait motion whose trunk orientations, arm motion, foot paths, and pelvis translations are similar to the patient's normal gait motion. Rather than varying control torque inputs and predicting motion outputs using forward dynamics, our optimizations varied motion (and ground reaction) inputs and predicted control torque outputs using inverse dynamics [26]. In this way, new gait motions could be predicted using stable inverse rather than unstable forward dynamic simulations for each function evaluation. 
The design variables for the one-cycle gait optimizations were coefficients defining the shape of each motion and ground reaction input curve. To develop initial guesses, we fitted each nominal input curve as a function of time using a combination of a cubic polynomial and eight Fourier harmonics (i.e., 20 coefficients per curve). RMS errors between experimental and fitted curves were $0.26 \mathrm{~mm}$ for joint translations, $0.17^{\circ}$ for joint rotations, $4.1 \mathrm{~N}$ for ground reaction forces, and $0.75 \mathrm{Nm}$ for ground reaction torques. Differentiating the polynomial-Fourier motion curves twice with respect to time produced the joint velocities and accelerations needed for inverse dynamic analyses. During each optimization, the following motion and ground reaction curves were varied by changing their polynomial-Fourier coefficients: pelvis superior/inferior translation, all back, pelvis, hip, knee, and ankle rotations, and all ground reaction forces and torques (i.e., 29 curves for a total of 580 design variables). Ground reactions were set to zero for time frames when the foot was known to be off the floor. Coefficients for shoulder and elbow rotations and pelvis horizontal translations were not varied so as to match the nominal gait motion. Walking speed was not varied by the dynamic optimizations.

We incorporated these design variables into an optimization cost function (1) that minimized the left and right knee adduction torques subject to several "reality" constraints implemented via a penalty method and consistent with our "normal looking" gait requirement

$$
\begin{aligned}
\min _{p} \sum_{i=1}^{101}\left[w_{1} \sum_{k=1}^{2}\left(T(p)_{\text {Add }}^{2}\right)_{i k}\right. \\
\quad+w_{2} \sum_{j=1}^{6} \sum_{k=1}^{2}\left(\Delta T(p)_{\text {Leg }}^{2}\right)_{i j k} \\
\quad+w_{3} \sum_{j=1}^{2} \sum_{k=1}^{2}\left(\Delta C o P(p)_{\text {Foot }}^{2}\right)_{i j k} \\
\quad+w_{4} \sum_{j=1}^{6} \sum_{k=1}^{2}\left(\Delta q(p)_{\text {Foot }}^{2}\right)_{i j k} \\
\quad+w_{5} \sum_{j=1}^{3}\left(\Delta q(p)_{\text {Trunk }}^{2}\right)_{\mathrm{ij}} \\
\left.\quad+w_{6} \sum_{j=1}^{3}\left(\Delta F(p)_{\text {Pelvis }}^{2}+\Delta T(p)_{\text {Pelvis }}^{2}\right)_{\mathrm{ij}}\right]
\end{aligned}
$$

where

$$
\begin{array}{ll}
p & \begin{array}{l}
\text { vector of } 580 \text { design variables } \\
\text { (polynomial-Fourier coefficients); }
\end{array} \\
i & \text { time frame (1 through 101); } \\
j & \begin{array}{l}
\text { joint translational or rotational axis (1 through } \\
2,3, \text { or } 6) ;
\end{array} \\
k & \text { side (1 through } 2) ; \\
w_{1} & \text { through } w_{6} \text { are cost function weights; } \\
T_{\text {Add }} & \text { knee adduction torque; }
\end{array}
$$

$\Delta T_{\text {Leg }} \quad$ change in a hip, knee, or ankle control torque away from its nominal value;

$\triangle C o P_{\text {Foot }}$ change in a center pressure location away from its nominal value measured with respect to the foot frame;

$\Delta q_{\text {Foot }} \quad$ change in a foot translation or rotation away from its nominal value measured with respect to the lab frame (these quantities are not DOFs in the model);

$\Delta q_{\text {Trunk }} \quad$ change in a trunk rotation away from its nominal value measured with respect to the lab frame (these quantities are not DOFs in the model);

$\Delta F_{\text {Pelvis }} \quad$ change in a residual pelvis force from its nominal value (close to zero) expressed in the lab frame;

$\Delta T_{\text {Pelvis }} \quad$ change in a residual pelvis torque away from its nominal value (close to zero) expressed in the lab frame.

Units for the various cost function terms were $\mathrm{mm}$ for translations, deg for rotations, $N$ for forces, and $\mathrm{Nm}$ for torques. The penalty terms (i.e., the terms with weight factors $w_{2}$ through $w_{6}$ ) forced the optimization to use muscle controls similar to the nominal case, keep the center of pressure under each foot, follow the nominal foot paths and trunk orientation, and eliminate external forces and torques acting on the pelvis. Since the cost function minimized a weighted sum of squares of errors, we again chose to use the Levenberg-Marquardt nonlinear least-squares algorithm in Matlab.

We performed two gait optimizations with this cost function, each with a different set of cost function weights. For both sets, one of the goals was for the nominal foot paths $\left(w_{4}\right)$, trunk orientation $\left(w_{5}\right)$, and residual pelvis loads $\left(w_{6}\right)$ to be tracked closely, similar to a constraint. We developed the first set of cost function weights by setting all weights to 1 and performing a trial optimization. We then systematically increased weights $w_{4}$ through $w_{6}$ by a factor of 10 until rms errors in the foot path, trunk orientation, and pelvis residual loads were all less than 1 . The resulting weights were 1 for $w_{1}$ through $w_{3}$ and 10 for $w_{4}$ through $w_{6}$. The second set of cost function weights started from the first set but added 50\% more weight to the adduction torque terms $\left(w_{1}\right)$ and $50 \%$ less weight to the loosely tracked control torque and center of pressure terms ( $w_{2}$ and $w_{3}$ ), thereby giving the optimizer greater freedom to reduce the adduction torque. The resulting weights were 1.5 for $w_{1}, 0.5$ for $w_{2}$ and $w_{3}$, and 10 for $w_{4}$ through $w_{6}$. The predictive gait optimizations were performed with the SIMM/Pipeline version of the model. Despite the use of 580 design variables, each optimization required only 45 min of CPU time on a 1.7-GHz Pentium M laptop.

\section{RESULTS}

Both optimizations predicted "normal looking" gait motions that significantly reduced both adduction torque peaks during left leg stance (Table I). SIMM animations of the predicted motions revealed a "medial-thrust" gait pattern that drove the left knee inward, causing the ground reaction force vector 
TABLE I

Summary of Optimized And PostTraining KNeE AdDuction TORQue Reductions Relative to Pretraining PeAK VAlues

\begin{tabular}{|c|c|c|c|c|c|c|}
\hline & \multicolumn{3}{|c|}{ First Peak } & \multicolumn{3}{|c|}{ Second Peak } \\
\hline & $\begin{array}{c}\text { Absolute } \\
\text { Value } \\
\left(\% W^{*} \mathrm{HT}\right)\end{array}$ & $\begin{array}{l}\text { Absolute } \\
\text { Reduction } \\
\left(\% W^{*} \mathrm{HT}\right)\end{array}$ & $\begin{array}{l}\text { Percent } \\
\text { Reduction } \\
(\%)\end{array}$ & $\begin{array}{c}\text { Absolute } \\
\text { Value } \\
\left(\% \mathrm{BW}^{*} \mathrm{HT}\right)\end{array}$ & $\begin{array}{l}\text { Absolute } \\
\text { Reduction } \\
\left(\% W^{*} H T\right)\end{array}$ & $\begin{array}{c}\text { Percent } \\
\text { Reduction } \\
(\%)\end{array}$ \\
\hline Pre-training & 3.8 & N/A & N/A & 4.6 & N/A & N/A \\
\hline Optimization tight & 2.6 & 1.2 & 32 & 3.0 & 1.6 & 34 \\
\hline Post-training moderate & 2.3 & 1.5 & 39 & 2.9 & 1.7 & 37 \\
\hline Optimization loose & 1.8 & 2.1 & 54 & 2.0 & 2.6 & 56 \\
\hline Post-training exaggerated & 1.9 & 1.9 & 50 & 2.1 & 2.5 & 55 \\
\hline
\end{tabular}
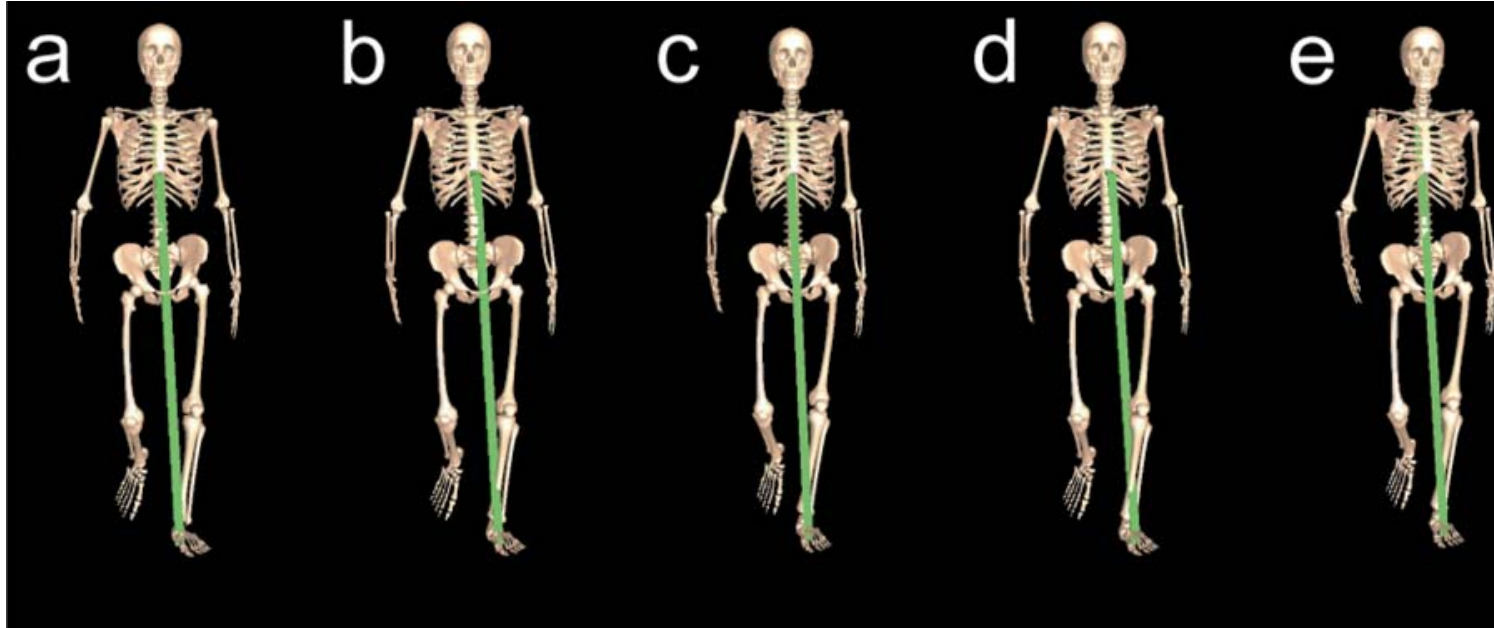

Fig. 2. Visualization of the moment arm of the ground reaction force vector about the knee center for experimental and optimized gait motions. (a) Nominal experimental gait motion. (b) Gait motion predicted using tighter tracking of leg control torques and center of pressure locations. (c) Gait motion measured posttraining when the patient tried to walk using his old gait pattern. (d) Gait motion predicted using looser tracking of leg control torques and center of pressure locations. (e) Gait motion measured posttraining when the patient tried to walk using an exaggerated version of the predicted gait motions. The selected time frame is the location of the first peak in the knee adduction torque curve.

to pass more laterally to the knee center than in the nominal experimental situation (Fig. 2). For the first set of cost function weights, the predicted adduction torque reductions were $1.2 \% \mathrm{BW} * \mathrm{HT}(32 \%)$ and $1.6 \% \mathrm{BW} * \mathrm{HT}(34 \%)$ in the first and second peaks, respectively, while for the second set, they were $2.1 \% \mathrm{BW} * \mathrm{HT}(54 \%)$ and $2.6 \% \mathrm{BW} * \mathrm{HT}(56 \%)$ (Fig. 3). The primary difference between the two sets of optimization results was that the second set predicted larger kinematic and kinetic changes to achieve larger adduction torque reductions.

The predicted medial-thrust gait motion involved three subtle kinematic changes: slightly decreased pelvis obliquity, slightly increased pelvis axial rotation, and slightly increased leg (i.e., simultaneous hip, knee, and ankle) flexion. During the stance phase, less pelvis obliquity created a more level pelvis while more pelvis axial rotation caused the stance leg hip to move anteriorly (Fig. 4). Coupled with a more flexed leg, these changes caused the stance leg knee to shift medially under the center of mass of the body. Most of the leg flexion increase came from the hip and knee, with a small amount coming from the ankle as well (Fig. 5). While the increases in hip, knee, and ankle flexion were proportional to the decrease in the knee adduction torque,
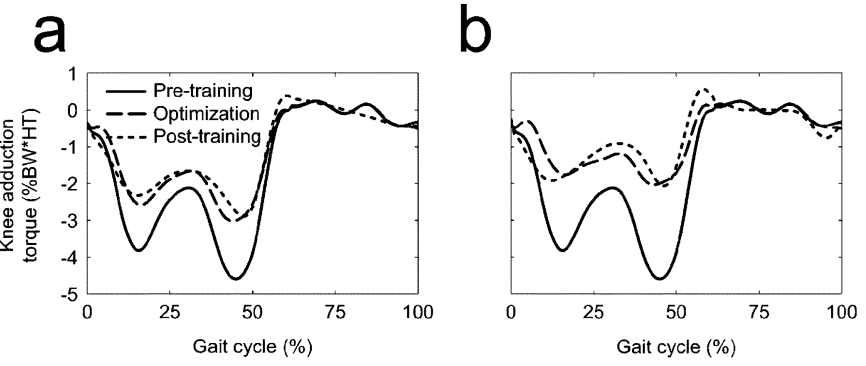

Fig. 3. Left knee adduction torque curves measured experimentally for the nominal gait motion (solid lines), predicted by the optimizations (dashed lines), and measured experimentally after gait retraining (dotted lines). (a) Results corresponding to tighter tracking of nominal experimental data. (b) Results corresponding to looser tracking of nominal experimental data.

the changes in pelvis rotations were approximately the same for both optimizations.

These kinematic changes were produced by three primary kinetic changes: decreased hip adduction torque, increased knee extension torque, and increased ankle inversion torque (Fig. 6). In all three cases, the magnitude of the change was proportional 


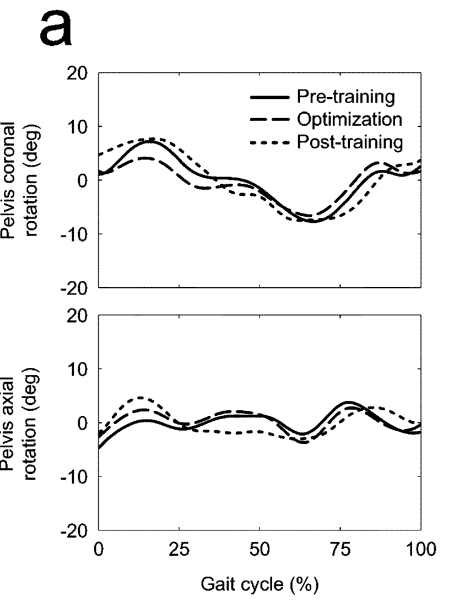

b
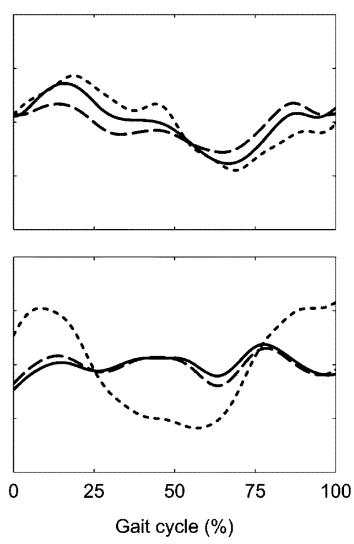

Fig. 4. Pelvis obliquity and axial rotation curves measured experimentally for the nominal gait motion (solid lines), predicted by the optimizations (dashed lines), and measured experimentally after gait retraining (dotted lines). (a) Results corresponding to tighter tracking of nominal experimental data. (b) Results corresponding to looser tracking of nominal experimental data.

\section{a}
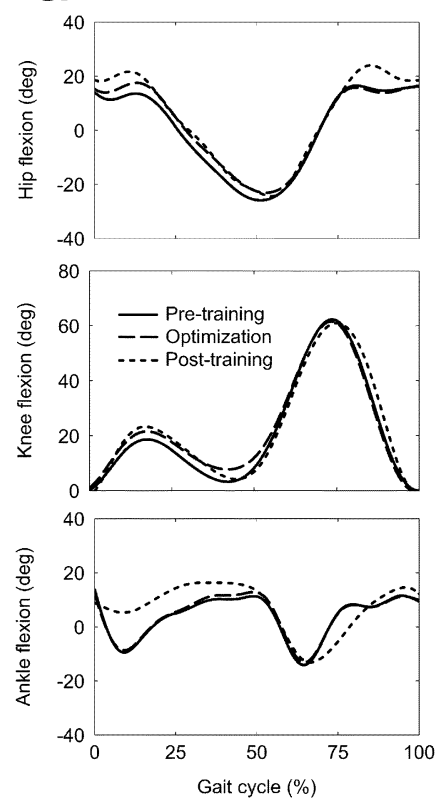

b
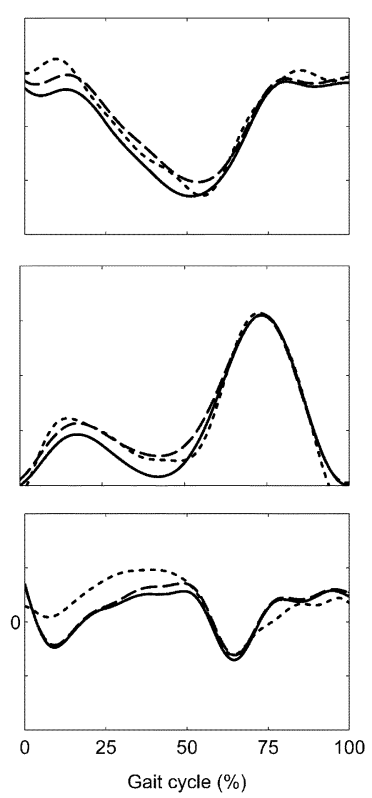

Fig. 5. Left hip, knee, and ankle flexion curves measured experimentally for the nominal gait motion (solid lines), predicted by the optimizations (dashed lines), and measured experimentally after gait retraining (dotted lines). (a) Results corresponding to tighter tracking of nominal experimental data. (b) Results corresponding to looser tracking of nominal experimental data.

to the magnitude of the adduction torque reduction. These joint torque changes resulted in a lateral shift of the center of pressure under the foot (Fig. 7) with little corresponding change in the ground reaction force (Fig. 8). The magnitude of the center of pressure change was also proportional to the magnitude of the adduction torque reduction. Kinetic changes predicted at the other joints were less prominent.

After gait retraining, the patient was able to achieve external knee adduction torque reductions comparable to the optimization predictions (Table 1; Figs. 2 and 3). When the a

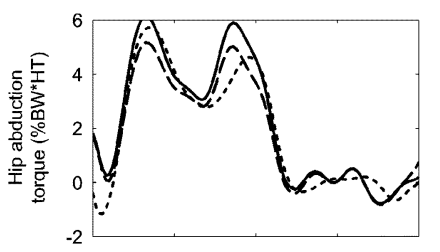

b
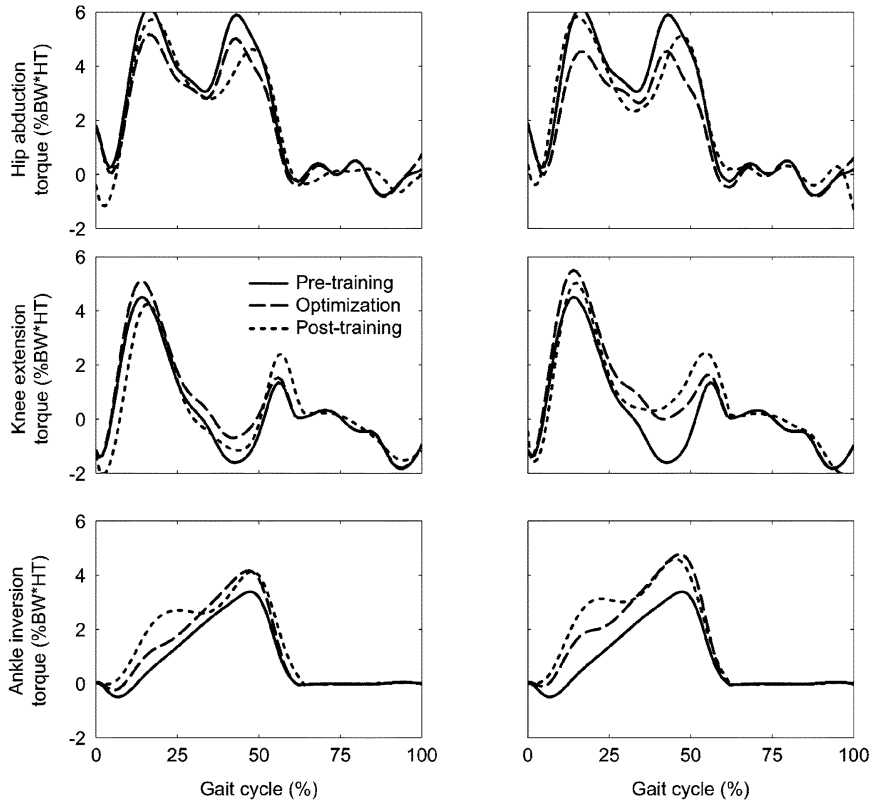

Fig. 6. Left hip abduction, knee extension, and ankle inversion torque curves measured experimentally for the nominal gait motion (solid lines), predicted by the optimizations (dashed lines), and measured experimentally after gait retraining (dotted lines). (a) Results corresponding to tighter tracking of nominal experimental data. (b) Results corresponding to looser tracking of nominal experimental data.

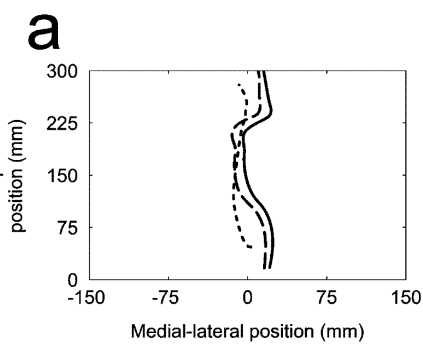

b

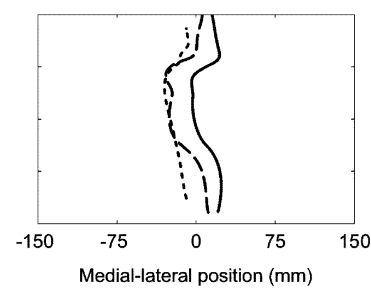

Fig. 7. Left foot center of pressure locations on the bottom of the foot measured experimentally for the nominal gait motion (solid lines), predicted by the optimizations (dashed lines), and measured experimentally after gait retraining (dotted lines). (a) Results corresponding to tighter tracking of nominal experimental data. (b) Results corresponding to looser tracking of nominal experimental data.

patient attempted to walk using his old gait pattern, he produced adduction torque reductions of $1.5 \% \mathrm{BW} * \mathrm{HT}(39 \%)$ and $1.7 \% \mathrm{BW} * \mathrm{HT}(37 \%)$ in the first and second peak, respectively. When he walked using an exaggerated version of the predicted medial-thrust gait motion, the reductions were $1.9 \% \mathrm{BW} * \mathrm{HT}$ (50\%) and $2.5 \% \mathrm{BW} * \mathrm{HT}(55 \%)$.

These decreases were achieved by kinematic and kinetic changes that were generally consistent with the optimization predictions (Figs. 4-7). However, several exceptions were noted. No decrease in pelvis obliquity and a larger than predicted increase in pelvis axial rotation were observed (Fig. 4). The increase in ankle flexion was also larger than predicted (Fig. 5). For kinetic quantities, the experimental decrease in hip adduction torque was generally less than predicted (Fig. 6). The increase in the first peak of the knee extension torque was 


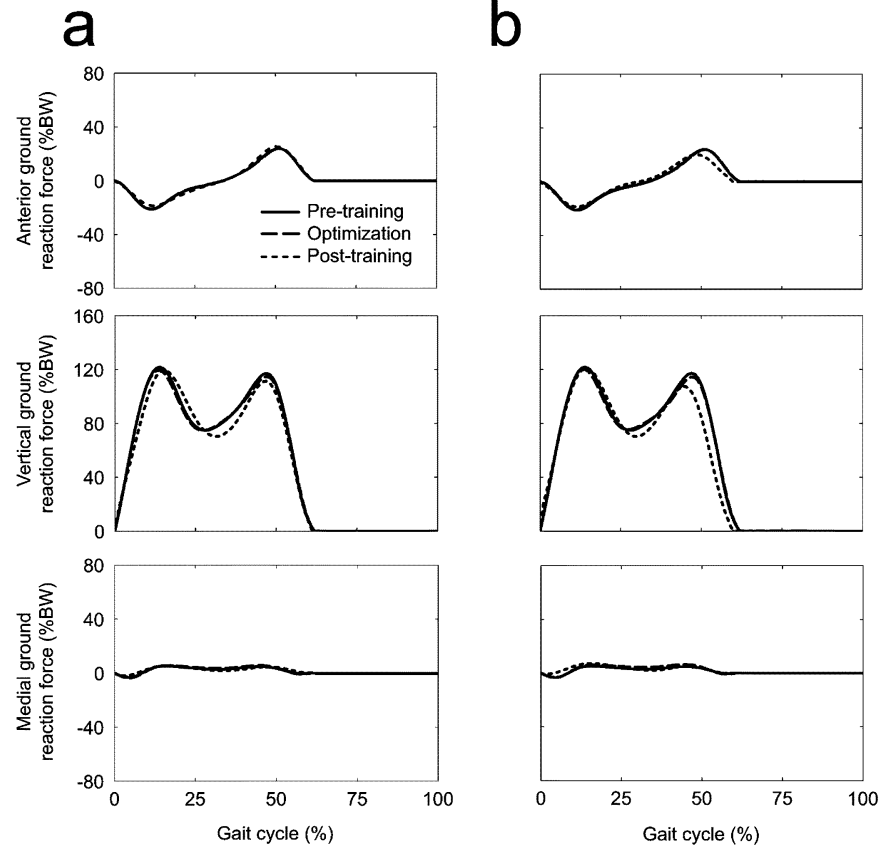

Fig. 8. Left foot ground reaction forces measured experimentally for the nominal gait motion (solid lines), predicted by the optimizations (dashed lines), and measured experimentally after gait retraining (dotted lines). (a) Results corresponding to tighter tracking of nominal experimental data. (b) Results corresponding to looser tracking of nominal experimental data.

smaller than predicted while the increase in the second peak was larger.

\section{DISCUSSION}

This study used dynamic optimization of a patient-specific, full-body gait model to predict 3-D gait modifications that reduce both peaks of the external knee adduction torque curve simultaneously. Two optimizations were performed starting from the patient's nominal pretreatment gait data - one with tighter and one with looser tracking of leg control torques and center of pressure. The predicted reductions of $32 \%$ to $56 \%$ in both peaks were extremely close to the decreases of $37 \%$ to $55 \%$ achieved by the patient after gait retraining. Furthermore, these decreases were comparable to the $30 \%$ to $50 \%$ observed following HTO surgery [3], [6], [9], which was the goal of the design process. The actual reductions achieved by the patient during normal walking would be somewhere within these ranges. To achieve these decreases, the optimizations predicted a "normal looking" gait motion that did not alter the foot path or trunk orientation. Instead, the optimizations medialized the knee by bringing it under the center of mass of the body while also shifting the center of pressure laterally under the foot. The synergistic 3-D kinematic changes needed for this strategy would have been difficult to discover by purely experimental means but were intuitive once identified by the optimizations. If generalizable to other patients with medial compartment knee OA, the predicted medial-thrust gait motion may provide a clinically useful rehabilitation strategy either apart from or in conjunction with HTO surgery (e.g., to reduce the chance of recurrent varus alignment [7], [28]).
The posttraining kinetic changes were generally consistent with the predicted strategy for lowering the knee adduction torque. Shifting the center of pressure laterally decreased the moment arm of the ground reaction force vector (applied at the center of pressure) around the hip center and knee center, resulting in a decreased hip abduction torque and external knee adduction torque. The minor discrepancy between the predicted and observed decrease in hip abduction torque can be attributed to the lack of decreased pelvis obliquity posttraining. When we performed an additional optimization that prescribed the pelvis obliquity to match the nominal gait motion, the predicted drop in hip abduction torque became similar to the posttraining observations, while the predicted drop in the knee adduction torque was only slightly less. In contrast to changes at the hip and knee, a lateral shift in the center of pressure increased the moment arm of the ground reaction force vector about the ankle center, necessitating increased ankle inversion torque. Medialization of the knee through a more flexed leg necessitated an increase in knee extension torque over much of stance phase. The lack of an increased first peak in knee extension torque for one of the posttraining cases is possibly due to the sensitivity of the knee extension torque calculation to small anterior-posterior errors in the knee center location.

Our inverse dynamics approach for performing full-body gait optimizations has advantages and disadvantages. One of the primary advantages is that the computational speed is already sufficient for use in a clinical environment. By replacing finite difference derivatives with automatic differentiation [29] in the Autolev version of the code, we can now perform full-body gait optimizations in approximately $10 \mathrm{~min}$ of CPU time. If unique gait modifications are needed for different patients, this short computation time makes it practical to customize treatment planning for individual patients. One of the main disadvantages is the presence of residual loads on the pelvis. These loads would not be present with a forward dynamics approach. When we predict new gait motions using inverse dynamics, the optimization uses the last penalty term in (1) to keep these residual loads at the same levels as in the pretraining experimental data set. Though we are not able to reduce the residual loads to zero, our results suggest that they do not adversely affect our predictive capabilities.

Treating the knee as a simple pin joint was the most significant modeling assumption in our computational methodology. The direction of our best-fit pin joint axis accounted for the average values of knee adduction-abduction and internal-external rotation produced by the patient during gait. Given noise due to skin movement artifacts, it would be difficult to determine accurate limits on these secondary joint motions. To minimize the effect of the pin joint assumption on our optimization predictions, we used a bottom-up inverse dynamics approach without joint constraints to calculate the nominal experimental adduction torque curve. We then determined how to match this curve using our full-body gait model with joint constraints. The critical factor was to make each shank segment in the full-body model match its experimental pose throughout stance phase. Thus, by placing large weight on shank marker coordinate errors during the full-body model calibration process, we were able to match the knee adduction torque curve produced by a model 
without joint constraints. Since the predicted adduction torque reductions were comparable to those observed experimentally, we do not believe that our pin joint knee assumption adversely affected our prediction process.

Another assumption in our computational methodology was that the selected cost function weights were representative of the patient's control strategy. Though we followed a systematic approach for selecting two sets of cost function weights, different weights will produce different optimization results. As in any engineering design study, the main goal is to achieve a final design that is better than the nominal one, whether or not the final design is the best one possible. Since the predicted gait modifications did, in fact, result in significant adduction torque reductions when implemented, this limitation is not a serious one.

The biggest limitation of our study was the evaluation of only a single patient. Since the goal of the study was to assess the feasibility of using dynamic optimization of a patient-specific gait model to design a patient-specific treatment, we believe that use of a single patient was reasonable. If we were unable to demonstrate that the methodology works for at least one patient, there would be little motivation for studying a larger number of patients. Our next step will be to evaluate whether the same methodology will work for other patients with knee OA. These patients will have different nominal gait patterns, adduction torque peaks, varus malalignment, and lateral collateral ligament laxity, all of which may affect the effectiveness of our model-based treatment approach.

Neural adaptation may explain why the subject achieved reduced knee adduction torque peaks posttraining when trying to walk with his pretraining gait pattern. The goal of gait retraining was for the subject to make the new gait pattern second nature. When the subject tried to walk posttraining with his pretraining gait pattern, he attempted to give his knees a slight lateral thrust. However, the review of video data revealed that he still exhibited increased medialization of the knees compared to pretraining. Consequently, the subject's adduction torque peaks did not reach pretraining levels, possibly because he had ingrained the new gait pattern through the retraining process. At least two previous studies have shown that gait retraining can produce sustained changes in gait mechanics [30], [31]. Sustained changes are also common in athletes, who make a conscious effort to modify their mechanics during training so that they will perform better during competition.

A related issue is how to perform gait retraining for future patients. The patient used in our study was highly knowledgeable on gait mechanics and so was able to understand and implement the necessary gait modifications by studying plots and animations of the optimization results. The nine-month retraining period was a function of the amount of time required to obtain access to a gait lab for retesting rather than the amount of time needed to learn and assimilate the new gait pattern. For other patients to learn the medial-thrust gait pattern and make it second nature, some type of real-time feedback coupled with a systematic retraining process, as recently performed successfully for tibial shock reduction [31], will likely be necessary.

A final clinical implementation issue is whether the proposed medial-thrust gait motion will result in detrimental loading changes at other joints. After a year and a half of walking with the medial-thrust gait pattern, the patient involved in our study has experienced reduced knee pain without any problems at other joints. Apart from the knee adduction torque, the largest load changes were in the internal ankle inversion and knee extension torque, both of which increased. For the nonexaggerated posttraining gait motion, the $25 \%$ increase in peak ankle inversion torque is comparable to that caused by $6^{\circ}$ lateral heel wedges [18]. Thus, the medial-thrust gait pattern is likely to be no worse for the ankle than it is for the introduction of lateral heel wedges. Furthermore, ankle OA is rare in clinical practice and is usually the result of previous traumatic injury [32], [33].

Though both knee extension torque peaks also increased posttraining, these increases do not necessarily imply an increase in medial contact force. First, little correlation exists between the magnitude of the knee extension torque and the magnitude of in vivo medial contact force measured by an instrumented knee replacement during gait [34]. Second, the largest quadriceps forces do not necessarily correspond to periods of the largest knee extension torque [35]. Third, patients with knee OA exhibit increased quadriceps-hamstrings co-contraction compared to normal subjects [36], suggesting that increased knee extension torque could be created by decreased hamstrings co-contraction, leading to a reduced medial contact force. Fourth, knee extensor strength training has been reported to decrease rather than increase pain in patients with knee OA [37]. Finally, our patient reduced both knee adduction torque peaks by 1.5 to $2.5 \% \mathrm{BW} * \mathrm{HT}$, which is highly significant as the risk of OA progression increases by a factor of 6.5 for each $1 \% \mathrm{BW} * \mathrm{HT}$ increase in peak knee adduction torque [12]. Given these observations for the ankle and knee, we believe that clinical evaluation of the generalizability, efficacy, and safety of the medial-thrust gait pattern in a small patient population is a logical next step.

\section{REFERENCES}

[1] O. D. Schipplein and T. P. Andriacchi, "Interaction between active and passive knee stabilizers during level walking," J. Orthop. Res., vol. 9 , pp. 113-119, 1991.

[2] T. P. Andriacchi, "Dynamics of knee malalignment," Orthop. Clin. North Am., vol. 25, pp. 395-403, 1994.

[3] M. Wada, S. Imura, K. Nagatani, H. Baba, S. Shimada, and S. Sasaki, "Relationship between gait and clinical results after high tibial osteotomy," Clin. Orthop. Relat. Res., vol. 354, pp. 180-188, 1998.

[4] D. E. Hurwitz, A. B. Ryals, J. P. Case, J. A. Block, and T. P. Andriacchi, "The knee adduction moment during gait in subjects with knee osteoarthritis is more closely correlated with static alignment than radiographic disease severity, toe out angle and pain," J. Orthop. Res., vol. 20, pp. 101-107, 2002.

[5] A. Mündermann, C. O. Dyrby, and T. P. Andriacchi, "Secondary gait changes in patients with medial compartment knee osteoarthritis," Arthritis Rheum., vol. 52, pp. 2835-2844, 2005.

[6] C. C. Prodromos, T. P. Andriacchi, and J. O. Galante, "The relationship between gait and clinical changes following high tibial osteotomy," $J$. Bone Joint Surg., vol. 67A, pp. 1188-1194, 1985.

[7] J. W. Wang, K. N. Kuo, T. P. Andriacchi, and J. O. Galante, "The influence of walking mechanics and time on the results of proximal tibial osteotomy," J. Bone Joint Surg., vol. 72A, pp. 905-913, 1990.

[8] J. M. Bryan, D. E. Hurwitz, B. R. Bach, T. Bittar, and T. P. Andriacchi, "A predictive model of outcome in high tibial osteotomy," in Proc. 43rd Annu. Meeting Orthop. Res. Soc., 1997, p. 718.

[9] L. Weidenhielm, O. K. Svensson, and L.-A. Broström, "Surgical correction of leg alignment in unilateral knee osteoarthritis reduces the load on the hip and knee bilaterally," Clin. Biomech., vol. 10, pp. 217-221, 1995.

[10] D. E. Hurwitz, D. R. Sumner, T. P. Andriacchi, and D. A. Sugar, "Dynamic knee loads during gait predict proximal tibial bone distribution," J. Biomech., vol. 31, pp. 423-430, 1998. 
[11] L. Sharma, D. E. Hurwitz, E. J.-M. A. Thonar, J. A. Sum, M. E. Lenz, D. D. Dunlop, T. J. Schnitzer, G. Kirwan-Mellis, and T. P. Andriacchi, "Knee adduction moment, serum hyaluronan level, and disease severity in medial tibiofemoral osteoarthritis," Arthritis Rheum., vol. 41, pp. 1233-1240, 1998.

[12] T. Miyazaki, M. Wada, H. Kawahara, M. Sato, H. Baba, and S. Shimada, "Dynamic load at baseline can predict radiographic disease progression in medial compartment knee osteoarthritis," Ann. Rheum. Dis., vol. 61, pp. 617-622, 2002.

[13] M. Andrews, F. R. Noyes, T. E. Hewett, and T. P. Andriacchi, "Lower limb alignment and foot angle are related to stance phase knee adduction in normal subjects: A critical analysis of the reliability of gait analysis data," J. Orthop. Res., vol. 14, pp. 289-295, 1996.

[14] M. Guo, M. J. Axe, and K. Manal, "The Influence of foot progression angle on the knee adduction moment during walking and stair climbing in pain free individuals with knee osteoarthritis," Gait Posture (in press).

[15] A. Mündermann, C. O. Dyrby, D. E. Hurwitz, L. Sharma, and T. P. Andriacchi, "Potential strategies to reduce medial compartment loading in patients with knee osteoarthritis of varying severity," Arthritis Rheum., vol. 50, pp. 1172-1178, 2004.

[16] J. L. DeMarre, L. Mündermann, L., T. P. Andriacchi, and A. Mündermann, "A mechanism to lower the knee adduction moment during walking: Gait retraining as intervention for knee OA," presented at the 30th Annu. Meeting Am. Soc. Biomech., 2006, paper \#173.

[17] W. Kakihana, M. Akai, N. Yamasaki, T. Takashima, and K. Nakazawa, "Changes in joint moments in the gait of normal subjects wearing laterally wedged insoles," Am. J. Phys. Med. Rehab., vol. 83, pp. 273-278, 2004.

[18] W. Kakihana, M. Akai, K. Nakazawa, T. Takashima, K. Naito, and S. Torii, "Effects of laterally wedged insoles on knee and subtalar joint moments," Arch. Phys. Med. Rehab., vol. 86, pp. 1465-1471, 2005.

[19] J. A. Reinbolt, J. F. Schutte, B. J. Fregly, B. I. Koh, R. T. Haftka, A. D. George, and K. H. Mitchell, "Determination of patient-specific multijoint kinematic models through two-level optimization," J. Biomech., vol. 38, pp. 621-626, 2005.

[20] F. C. Anderson and M. G. Pandy, "Dynamic optimization of human walking," J. Biomech. Eng., vol. 123, pp. 381-390, 2001.

[21] A. J. Van den Bogert, G. D. Smith, and B. M. Nigg, "In vivo determination of the anatomical axes of the ankle joint complex: An optimization approach,” J. Biomech., vol. 27, pp. 1477-1488, 1994.

[22] F. C. Anderson and M. G. Pandy, "A dynamic optimization solution for vertical jumping in three dimensions," Comput. Meth. Biomech. Biomed. Eng., vol. 2, pp. 201-231, 1999.

[23] R. R. Neptune, I. C. Wright, and A. J. Van den Bogert, "A method for numerical simulation of single limb ground contact events: Application to heel-toe running," Comput. Meth. Biomech. Biomed. Eng., vol. 3, pp. 321-334, 2001

[24] Z. Popovic, "Motion transformation by physically based spacetime optimization," Ph.D. dissertation, Dept. Comput. Sci., Carnegie Mellon Univ., Pittsburgh, PA, 1999.

[25] L. Chéze, B. J. Fregly, and J. Dimnet, "Determination of joint functional axes from noisy marker data using the finite helical axis," Hum. Mov. Sci., vol. 17, pp. 1-15, 1998.

[26] M. L. Nagurka and V. Yen, "Fourier-based optimal control of nonlinear dynamic systems," J. Dyn. Syst. Meas. Cont., vol. 112, pp. 17-26, 1990.

[27] P. D. Leva, "Adjustments to Zatsiorsky-Seluyanov's segment inertia parameters," J. Biomech., vol. 29, pp. 1223-1230, 1996.

[28] G. Magyar, S. Toksvig-Larsen, and A. Lindstrand, "Changes in osseous correction after proximal tibial osteotomy," Acta. Orthop. Scand., vol. 70, pp. 473-477, 1999 .
[29] A. Griewank, D. Juedes, and J. Utke, "ADOL-C: A package for the automatic differentiation of algorithms written in C/C++," Assoc. Comput. Mach. Trans. Math. Software, vol. 22, pp. 131-167, 1996.

[30] J. Schröter, V. Güth, M. Overbeck, D. Rosenbaum, and W. Winkelmann, "The 'Entlastungsgang'. A hip unloading gait as a new conservative therapy for hip pain in the adult," Gait Posture, vol. 9, pp. $151-157,1999$.

[31] H. P. Crowell and I. S. Davis, I.S., "Reducing lower extremity loads through gait retraining using real-time feedback methods," in Proc. 30th Annu. Meeting Am. Soc. Biomech., 2006, paper \#318.

[32] J. A. Buckwalter, C. Saltzman, and T. Brown, "The impact of osteoarthritis: Implications for research," Clin. Orthop. Rel. Res., vol. 427, pp. S6-S15, 2004.

[33] C. L. Saltzman, M. L. Salamon, G. M. Blanchard, T. Huff, A. Hayes, J. A. Buckwalter, and A. Amendola, "Epidemiology of ankle arthritis: Report of a consecutive series of 639 patients from a tertiary orthopaedic center," Iowa Orthop. J., vol. 24, pp. 44-46, 2004.

[34] D. Zhao, S. A. Banks, K. H. Mitchell, D. D. D'Lima, C. W. Colwell, and B. J. Fregly, "Correlation between the knee adduction torque and medial contact force for a variety of gait patterns," J. Orthop. Res., vol. 25, pp. 789-797, 2007.

[35] K. Manal, J. Gardinier, and N. Chimera, "What are we missing when using inverse dynamics," presented at the 30th Annu. Meeting Am. Soc. Biomech., 2006, paper \#323.

[36] T. Hortobagyi, L. Westerkamp, S. Beam, J. Moody, J. Garry, D. Holbert, and P. DeVita, "Altered hamstring-quadriceps muscle balance in patients with knee osteoarthritis," Clin. Biomech., vol. 20, pp. 97-104, 2005.

[37] L. A. Talbot, J. M. Gaines, S. M. Ling, and E. J. Metter, "A homebased protocol of electrical muscle stimulation for quadriceps muscle strength in older adults with osteoarthritis of the knee," J. Rheum., vol. 30, pp. 1571-1578, 2003

Benjamin J. Fregly, photograph and biography not available at the time of publication.

Jeffrey A. Reinbolt, photograph and biography not available at the time of publication.

Kelly L. Rooney, photograph and biography not available at the time of publication.

Kim H. Mitchell, photograph and biography not available at the time of publication.

Terese L. Chmielewski, photograph and biography not available at the time of publication. 